\title{
ANALYSIS OF QUADRATURE AND FREQUENCY SPLIT IN A MEMS VIBRATING RING GYROSCOPE WITH STRUCTURAL IMPERFECTIONS
}

Mehran Hosseini-Pishrobat ${ }^{1}$ and Erdinc Tatar ${ }^{1,2}$

${ }^{1}$ Department of Electrical and Electronics Engineering, Bilkent University, Ankara, TURKEY

${ }^{2}$ National Nanotechnology Research Center (UNAM), Bilkent University, Ankara, TURKEY

\begin{abstract}
Structural imperfections affect the performance of MEMS vibrating ring gyroscopes (VRGs), dominantly in terms of quadrature error and frequency split. We consider a VRG with a ring subjected to width nonuniformity and supported by an imperfect suspension. This reflects the scenario of nonuniform etching, common in microfabrication processes. We show that the ring's width nonuniformity mainly results in a rotation of the mode-shapes with respect to those of a perfect ring while an imperfect suspension induces frequency split between the modes. On this basis, we calculate the quadrature error in the gyroscope's output. As an example, a $4^{\circ}$ modeshape rotation and $15 \mathrm{~Hz}$ frequency split in a $60 \mathrm{kHz}$ VRG generate an approximate quadrature error of $1000^{\circ} \mathrm{s}$.
\end{abstract}

\section{KEYWORDS}

Frequency split, MEMS gyroscope, Quadrature error, Vibrating ring

\section{INTRODUCTION}

Vibrating ring gyroscopes (VRGs) are a class of Coriolis-based MEMS gyroscopes that use rings as the main vibrating element. A distinguishing feature of these gyroscopes is that a ring, because of its circular symmetry, inherently provides two orthogonal vibrating modes, which occur as degenerate pairs [1]. For an ideal ring with a uniform cross-section and isotropic material, these mode-shapes have the same natural frequency and their location is indeterminate [2]. That is, one can generate the mode-shapes at any desired location by a suitable actuation configuration. However, the presence of any geometric nonuniformity and/or material anisotropy renders the location of the mode-shapes determinate. In such a case, any misalignment between the actuation/sensing directions and the mode-shapes' principal axes leads to undesired couplings, deteriorating the Coriolis response. These couplings manifest themselves mainly as quadrature errors in the gyroscope's output, which can completely mask the Coriolis response. In addition, any deviation from the ideal ring can produce a frequency split between the modes. These factors significantly influence the performance of VRGs and hence, need to be studied.

The problem of imperfections in rings, either in the context of MEMS gyroscopes or mechanical vibration, has been studied in the literature. Laura et al. [3] used the Rayleigh-Ritz and finite element methods to estimate the natural frequencies of a ring with varying cross-section. Gallacher et al. [2] considered a VRG with couplings terms in its mass and stiffness matrices and presented a compensation method based on electrostatic spring softening. Bisegna and Caruso [4] presented a perturbation theory-based analysis for the natural frequencies and mode-shapes of imperfect rings. A similar line of work was pursued in [5] to study the frequency split and vibration instability in a ring subjected to angular velocities. The link between geometric defects and imperfections in the ring vibration, however, has not been investigated nor incorporated in these works.

The main goal of this paper is to present an analysis for the frequency split and quadrature error in the VRGs subjected to geometric nonidealities. Due to imperfections of microfabrication processes, such nonidealities are inevitable in practical gyroscopes. As a prime example, nonuniform etching across a wafer results in a non-perfect mechanical structure with undercuts (i.e., non-perfect sidewall angles) and width nonuniformities. Accordingly, as the main novelty, we consider a VRG whose ring has a nonuniform width, and it is also held by an imperfect inner suspension. In terms of vibrational properties, these imperfections can bring about off-diagonal terms in the mass and stiffness matrices. Hence, our analysis is based on calculating the kinetic and stiffness energies of the gyroscope to obtain these matrices.

Notation: Throughout this paper, [.] $]^{\top}$ stands for the matrix transpose.

\section{IMPERFECTION ANALYSIS}

Figure 1 shows the mechanical model of the VRG which is currently under development by our research group. The main vibrating element of the gyroscope is a ring having the mean radius, $R$ and the thickness, $h$. The ring is surrounded, both inside and outside, by capacitive actuation and sensing electrodes. To analyze the gyroscope's dynamics, we consider the cylindrical coordinate system $\{r, \theta, z\}$ whose origin coincides with the ring's center. The ring is supported by $N$ beams that connect it to an inner suspension at the angular positions, $\theta_{i}, i=1: N$. The whole structure is made of a material with the mass density, $\rho$ and Young's modulus, $E$. We consider a nonuniform ring width profile, $w(\theta), \theta \in$ $[0,2 \pi)$, which has its minimum $w_{\min }$ at $\theta=\phi \in[0, \pi]$, its maximum $w_{\max }$ at $\theta=\phi+\pi$, and varies linearly between these two extremes as shown in Figure 2. In our analysis, we adhere to the narrow ring and inextensibility assumptions [6]. Following the first assumption, we consider the displacement field $\left[u_{r}(\theta, t), u_{\theta}(\theta, t)\right]^{\top}$ defined at the ring's centerline to describe the in-plane motion of the structure; the subscripts $r$ and $\theta$ denote the displacements in the radial and tangential directions, respectively, and $t$ is time. Owing to the circular symmetry of the ring, its mode-shapes occur as the degenerate pairs, $\{\cos (n \theta), \sin (n \theta)\}, n=1,2, \ldots$ [7]. Therefore, taking into account inextensibility, the displacement field for the $n$-th mode is given by 


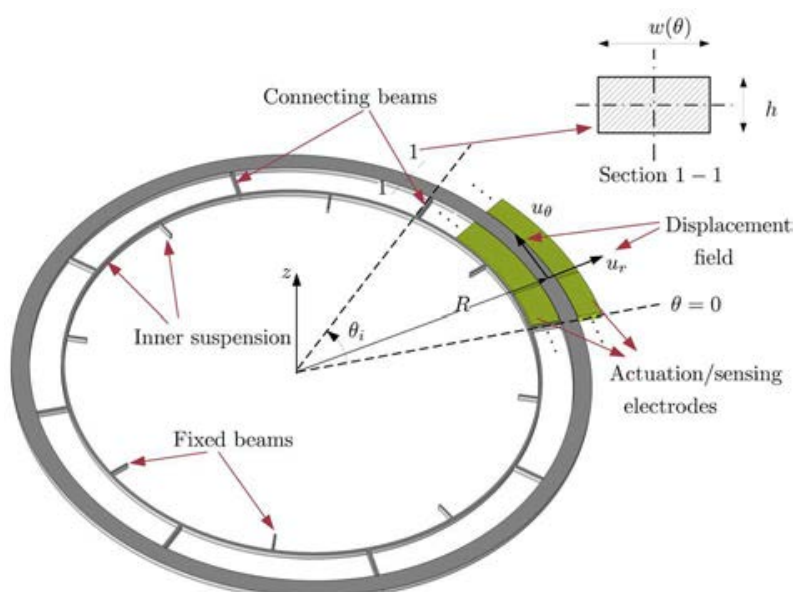

Figure 1: Schematics of the vibrating ring gyroscope.

$u_{\theta}(\theta, t)=-Q_{1}(t) \frac{\sin (n \theta)}{n}+Q_{2}(t) \frac{\cos (n \theta)}{n}$,

$u_{r}(\theta, t)=Q_{1}(t) \cos (n \theta)+Q_{2}(t) \sin (n \theta)$,

where $Q_{1}($.$) and Q_{2}($.$) are the generalized coordinates$ corresponding to the contributions of $\cos (n \theta)$ and $\sin (n \theta)$ mode-shapes to the displacement field. The generalized coordinates define a new Cartesian coordinate system, $\left\{Q_{1}, Q_{2}\right\}$ to analyze the ring's motion.

Given the displacement field (1), we obtain the vibrational properties of the ring by calculating its kinetic and potential energies.

Kinetic energy: Let us assume that the gyroscope is subjected to a constant angular velocity $\Omega_{z}$ about the $z$ axis. Assuming negligible linear accelerations, the velocity field of the ring is given by $\left[v_{r}(\theta, t), v_{\theta}(\theta, t)\right]^{\top}$, where

$v_{r}(\theta, t)=\dot{u}_{r}(\theta, t)-\Omega_{z} u_{\theta}(\theta, t)$,

$v_{\theta}(\theta, t)=\dot{u}_{\theta}(\theta, t)+\Omega_{z}\left(u_{r}+R\right)$.

By neglecting the centripetal terms, we calculate the kinetic energy as [8]

$\mathcal{T}=\frac{1}{2} \rho R h \int_{0}^{2 \pi}\left(v_{r}^{2}(\theta, t)+v_{\theta}^{2}(\theta, t)\right) w(\theta) \mathrm{d} \theta$

$\approx \frac{1}{2} \dot{Q}^{\top} M \dot{Q}+\Omega_{z} Q^{\top} C \dot{Q}$,

where $Q:=\left[Q_{1}, Q_{2}\right]^{\top}, M$ is the symmetric mass matrix, and $C$ is a skew-symmetric matrix associated with the Coriolis force. After substituting (2) into (3), we obtain these matrices as

$M=\left[\begin{array}{ll}m_{11} & m_{12} \\ m_{12} & m_{22}\end{array}\right], C=\left[\begin{array}{cc}0 & c_{12} \\ -c_{12} & 0\end{array}\right]$,

$m_{11}=\frac{\rho R h}{2 n^{2}} \int_{0}^{2 \pi}\left\{n^{2}+1+\left(n^{2}-1\right) \cos (2 n \theta)\right\} w(\theta) \mathrm{d} \theta$,

$m_{22}=\frac{\rho R h}{2 n^{2}} \int_{0}^{2 \pi}\left\{n^{2}+1-\left(n^{2}-1\right) \cos (2 n \theta)\right\} w(\theta) \mathrm{d} \theta$,

$m_{12}=\frac{\rho R h\left(n^{2}-1\right)}{2 n^{2}} \int_{0}^{2 \pi} \sin (2 n \theta) w(\theta) \mathrm{d} \theta$,

$c_{12}=\frac{\rho R h}{n} \int_{0}^{2 \pi} w(\theta) \mathrm{d} \theta$.

For the considered width profile, $w(\theta)$ we have

$m_{11}=m_{22}=m=\frac{\pi \rho R h\left(n^{2}+1\right)}{n^{2}}\left(\frac{w_{\min }+w_{\max }}{2}\right)$,

$m_{12}=0$,

$c_{12}=m_{c}=\frac{2 \pi \rho R h}{n}\left(\frac{w_{\min }+w_{\max }}{2}\right)$.

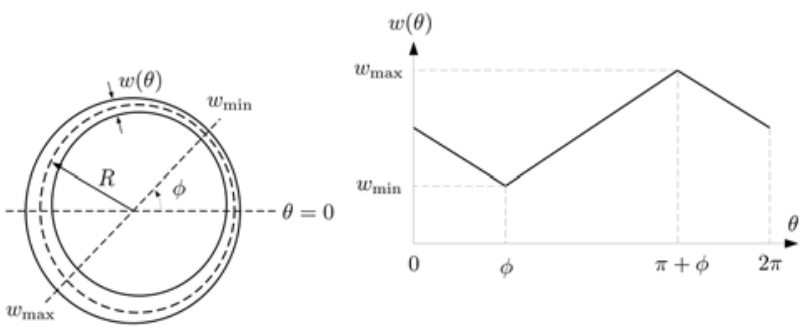

Figure 2: Nonuniform width profile of the ring.

Equation (5) indicates that the effective mass properties of the gyroscope are only affected by $w_{\text {min }}, w_{\text {max }}$ values of the width nonuniformity and not the angle, $\phi$. In addition, the symmetric nonunifomity, $w(\theta)$ induces no mass coupling between the mode-shapes as $m_{12}=0$.

Angular gain, denoted by AG, is an important parameter of VRGs that measures the efficiency of the gyroscope's structure in transferring momentum between its vibration modes via Coriolis coupling. More specifically, AG is the angular lag, due to inertia, between the rotation of the mode-shapes and the expected rotation angle, $\Omega_{z} t[1]$. For the $n$-th mode of the ring, AG is given by

$\mathrm{AG}=\frac{1}{n}\left(\frac{m_{c}}{m}\right)=\frac{2}{n^{2}+1}$.

Strain energy: From a mechanical vibration perspective, the inner suspension affects the ring in terms of inertia, damping, and stiffness. Because of the quasistatic state of the inner structure, stiffness is the dominant aspect of the ring-suspension interaction. The total potential energy of the gyroscope, $\mathcal{V}$ is the sum of strain energies $\mathcal{V}_{r}$ and $\mathcal{V}_{s}$ over the ring and the inner suspension, respectively. Following the Euler-Bernoulli beam theory, we only consider the strain energy due to the bending of the ring and neglect the effect of shear and axial stresses. We thereby have

$\mathcal{V}_{r}=\frac{E h}{24 R^{3}} \int_{0}^{2 \pi}\left(u_{r}(\theta, t)+\frac{\partial^{2} u_{r}(\theta, t)}{\partial \theta^{2}}\right)^{2} w^{3}(\theta) \mathrm{d} \theta$

$=\frac{1}{2} Q^{\top} K_{r} Q$,

where $K_{r}$ is the symmetric stiffness matrix of the ring. By substituting (1) into (7), we get

$K_{r}=\left[\begin{array}{ll}k_{11}^{r} & k_{12}^{r} \\ k_{12}^{r} & k_{22}^{r}\end{array}\right]$,

$k_{11}^{r}=\frac{E h}{24 R^{3}}\left(n^{2}-1\right)^{2} \int_{0}^{2 \pi}\{1+\cos (2 n \theta)\} w^{3}(\theta) \mathrm{d} \theta$,

$k_{22}^{r}=\frac{E h}{24 R^{3}}\left(n^{2}-1\right)^{2} \int_{0}^{2 \pi}\{1-\cos (2 n \theta)\} w^{3}(\theta) \mathrm{d} \theta$,

$k_{12}^{r}=\frac{E h}{24 R^{3}}\left(n^{2}-1\right)^{2} \int_{0}^{2 \pi} \sin (2 n \theta) w^{3}(\theta) \mathrm{d} \theta$.

For the considered width nonuniformity, $w(\theta)$ we have

$K_{r}=\left[\begin{array}{cc}k_{0}^{r}+k_{1}^{r} \cos (2 n \phi) & k_{1}^{r} \sin (2 n \phi) \\ k_{1}^{r} \sin (2 n \phi) & k_{0}^{r}-k_{1}^{r} \cos (2 n \phi)\end{array}\right]$,

$k_{0}^{r}=\frac{E h \pi\left(n^{2}-1\right)^{2}}{48 R^{3}}\left(w_{\text {max }}+w_{\text {min }}\right)\left(w_{\text {max }}^{2}+w_{\text {min }}^{2}\right)$,

$k_{1}^{r}=\frac{E h\left(n^{2}-1\right)^{2}}{16 \pi R^{3} n^{2}}\left(w_{\text {max }}+w_{\text {min }}\right)\left(w_{\text {max }}-w_{\text {min }}\right)^{2}$.

We model the effect of the inner suspension, at the angle $\theta_{i}$ of the $i$-th connecting beam, by the spring constant $k_{r_{i}}$ and $k_{\theta_{i}}$ along the radial and tangential directions, respectively. We further assume that the connecting beams are located at the nodal points of the $n$-th 
mode-i.e., $\theta_{i}=\frac{\pi}{2 n}(i-1), i=1: 4 n$. Accordingly, the potential energy of the suspension structure is given by

$\mathcal{V}_{s}=\sum_{i=1}^{N} \frac{1}{2} k_{r_{i}} u_{r}^{2}\left(\theta_{i}, t\right)+\frac{1}{2} k_{\theta_{i}} u_{\theta}^{2}\left(\theta_{i}, t\right)=\frac{1}{2} Q^{\top} K_{s} Q$,

where $K_{s}$ is the symmetric stiffness matrix of the suspension structure. By substituting the displacement filed (1) into (10), we obtain

$K_{s}=\left[\begin{array}{cc}k_{11}^{s} & 0 \\ 0 & k_{22}^{s}\end{array}\right]$,

$k_{11}^{s}=\sum_{i=1}^{2 n} k_{r_{2 i-1}}+\frac{k_{\theta_{2 i-1}}}{n^{2}}, k_{22}^{s}=\sum_{i=1}^{2 n} k_{r_{2 i}}+\frac{k_{\theta_{2 i}}}{n^{2}}$.

We note that for a perfect suspension, with identical connecting beams, $K_{S}$ reduces to

$k_{11}^{s}=k_{22}^{s}=k_{s}=2 n k_{r}+\frac{2 k_{\theta}}{n}$.

Since $\mathcal{V}=\mathcal{V}_{r}+\mathcal{V}_{s}$, the total stiffness matrix is given by

$K=K_{r}+K_{s}=$

$\left[\begin{array}{cc}k_{0}^{r}+k_{11}^{s}+k_{1}^{r} \cos (2 n \phi) & k_{1}^{r} \sin (2 n \phi) \\ k_{1}^{r} \sin (2 n \phi) & k_{0}^{r}+k_{22}^{s}-k_{1}^{r} \cos (2 n \phi)\end{array}\right]$.

We remark that the suspension's stiffness distribution becomes symmetric with respect the $n$-th mode by placing the identical connecting beams on the nodal points. Hence, in Equation (13), the off-diagonal terms are only caused by the ring's width nonuniformity. In addition, for a uniform width, we have $k_{1}^{r}=0$.

Upon calculating the mass and stiffness matrices, we study the following generalized eigenvalue problem:

$K v=\omega^{2} M v$.

The eigenvalues, $\omega^{2}$ give us the natural frequencies, and the eigenvectors, $v$ define the principal axes that determine the orientation of the mode-shapes with respect to our coordinate system. On this basis, we obtain the following results:

Case 1. Nonuniform width, perfect suspension:

1. In this case, the stiffness matrix, $K$ is a linear combination of the identity matrix and a rotation matrix associated with the angle, $2 n \phi$. On this basis, we obtain the natural frequencies as $\omega_{1,2}=\sqrt{\frac{k_{0}^{r}+k_{s} \pm k_{1}^{r}}{m}}$. Therefore, the frequency split, $\Delta \omega=\omega_{1}-\omega_{2}$ is independent of the angle, $\phi$ and it depends only on $w_{\min }$ and $w_{\max }$ parameters of the width nonuniformity. We note that, for a ring with uniform width, we have the single natural frequency $\omega_{0}=\sqrt{\frac{k_{0}^{r}+k_{s}}{m}}$ for both $\{\cos (n \theta)\}$ and $\{\sin (n \theta)\}$ mode-shapes. From Equation (9), we observe that $k_{1}$ is proportional to the second power of the width variation, $w_{\max }-w_{\min }$. Therefore, for small width variations, we have $\frac{k_{1}^{r}}{k_{0}^{r}+k_{s}^{r}} \ll 1$ and $\Delta \omega \approx \frac{k_{1}^{r}}{k_{0}^{r}+k_{s}} \omega_{0}$. Hence, small width variations have a negligible effect on the frequency split.

2. The principal axes $\left\{Q_{1}^{*}, Q_{2}^{*}\right\}$ are given by the following orthonormal (i.e., unit length and zero inner product) eigenvectors:

$v_{1}=\left[\frac{\sin (2 n \phi)}{\sqrt{2(1-\cos (2 n \phi))}}, \sqrt{\frac{1-\cos (2 n \phi)}{2}}\right]^{\top}$,
$v_{2}=\left[\frac{-\sin (2 n \phi)}{\sqrt{2(1+\cos (2 n \phi))}}, \sqrt{\frac{1+\cos (2 n \phi)}{2}}\right]^{\top}$.

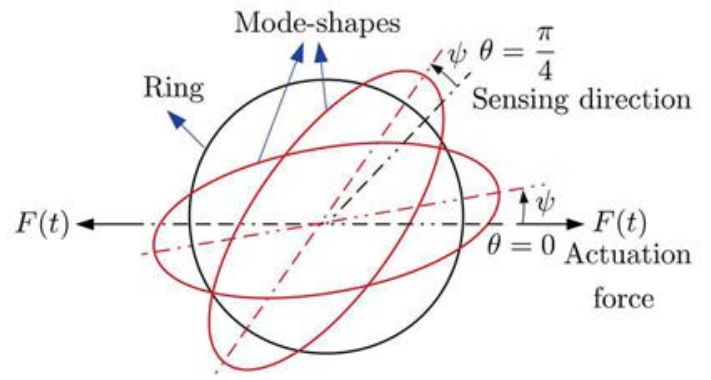

Figure 3: Rotation of $n=2$ mode-shapes of an imperfect ring.

The principal axes $\left\{Q_{1}^{*}, Q_{2}^{*}\right\}$ are rotated by an angle $\psi_{Q}$ with respect to the nominal $\left\{Q_{1}, Q_{2}\right\}$ axes. This translates into a rotation by the angle $\psi=\frac{\psi_{Q}}{n}$ of the mode-shapes $\{\cos (n \theta), \sin (n \theta)\}$ with respect to the reference axis, $\theta=0$. Using the properties of rotation matrices, we obtain

$\tan (n \psi)= \begin{cases}\tan (n \phi), & \text { if } \sin (2 n \phi)>0 \\ \tan \left(n \phi-\frac{\pi}{2}\right), & \text { if } \sin (2 n \phi)<0 .\end{cases}$

As a result, the rotation angle $\psi$ of the mode-shapes is independent of $w_{\min }, w_{\max }$ and depends solely on the angle, $\phi$ of the nonuniformity profile.

Case 2. Uniform width, imperfect suspension:

1. The natural frequencies are $\omega_{1}=\sqrt{\frac{k_{0}^{r}+k_{11}^{s}}{m}}$ and $\omega_{2}=$ $\sqrt{\frac{k_{0}^{r}+k_{22}^{S}}{m}} ;$ accordingly, we have the frequency split

$|\Delta \omega|=\left|\omega_{1}-\omega_{2}\right|=\frac{\left|k_{11}^{S}-k_{22}^{S}\right|}{\sqrt{m}\left(\sqrt{k_{0}^{r}+k_{11}^{S}}+\sqrt{k_{0}^{r}+k_{22}^{S}}\right)}$.

2. The principal axes are the same as the nominal $\left\{Q_{1}, Q_{2}\right\}$ axes and therefore, no mode-shape rotation is induced by the imperfect suspension.

\section{Quadrature error}

Consider the VRG shown in Figure 1 operating in the $n$-th vibration mode. We place the differential actuation and sensing electrodes at the extrema of $\cos (n \theta)$ and $\sin (n \theta)$, respectively. Because of imperfections, the mode-shapes of the ring are rotated by the angle $\psi$ with respect to $\theta=$ 0 ; that is, the mode-shapes are given by $\{\cos (n(\theta-$ $\psi)), \sin (n(\theta-\psi))\}$ with $\left\{Q_{1}^{*}, Q_{2}^{*}\right\}$ as the corresponding generalized coordinates. This rotation, in turn, generates misalignment between the designated actuation/sense directions and the ring's principal axes (see Figure 3). As a result, the gyroscope's operation deviates from the desired drive mode-sense mode setting where no actuation misalignment exists and the Coriolis force is the only coupling between the modes. We explain this point as follows.

Actuation: Assuming a driving force of the form $F(t)=$ $F_{0} \sin \left(\omega_{1} t\right)$, then its projection onto the $\{\cos (n(\theta-\psi))\}$ and $\{\sin (n(\theta-\psi))\}$ mode-shapes are given by $F_{0} \cos (n \psi) \sin \left(\omega_{1} t\right) \quad$ and $\quad F_{0} \sin (n \psi) \sin \left(\omega_{1} t\right)$, respectively. In the perfect case, $\psi=0$ and the actuation acts only on the $\{\cos (n \theta)\}$ mode and, thereby, it serves as the VRG's drive mode. For $\psi \neq 0$, however, there exists a projection of the actuation force onto the other mode. In the latter case, the VRG's dynamics is governed by 
$\left[\begin{array}{c}\ddot{Q}_{1}^{*} \\ \ddot{Q}_{2}^{*}\end{array}\right]+\left[\begin{array}{cc}\omega_{1} q_{f}^{-1} & 0 \\ 0 & \omega_{2} q_{f}^{-1}\end{array}\right]\left[\begin{array}{c}\dot{Q}_{1}^{*} \\ \dot{Q}_{2}^{*}\end{array}\right]+\left[\begin{array}{cc}\omega_{1}^{2} & 0 \\ 0 & \omega_{2}^{2}\end{array}\right]\left[\begin{array}{l}Q_{1}^{*} \\ Q_{1}^{*}\end{array}\right]$

$=\left[\begin{array}{c}F_{0} \cos (n \psi) \\ F_{0} \sin (n \psi)\end{array}\right] \sin \left(\omega_{1} t\right)-2 n A G \Omega_{z}\left[\begin{array}{cc}0 & -1 \\ 1 & 0\end{array}\right]\left[\begin{array}{c}\dot{Q}_{1}^{*} \\ \dot{Q}_{2}^{*}\end{array}\right]$,

where $q_{f}$ is the quality factor.

Sensing: Our capacitive measurement of the ring's motion is given by the average displacement

$\frac{1}{\theta_{e}} \int_{\frac{\pi}{2 n}-\frac{\theta_{e}}{2}}^{\frac{\pi}{2 n}+\frac{\theta_{e}}{2}} u_{r}(\theta, t) \mathrm{d} \theta=\frac{2 \sin \left(\frac{n \theta_{e}}{2}\right)}{n \theta_{e}}\left(\begin{array}{c}Q_{1}^{*} \sin (n \psi) \\ +Q_{2}^{*} \cos (n \psi)\end{array}\right)$,

where $\theta_{e}$ is the angular span of the electrodes. For the perfect ring $\psi=0$, the $\{\sin (n \theta)\}$ mode serves as the VRG's sense mode and, thereby, our measurement contains only $Q_{2}^{*}$ that contains the Coriolis response. However, in the imperfect case $\psi \neq 0$, there exists a projection of the drive mode's motion onto the measurement.

By solving the differential equation (18) and substituting it into the measurement (19), we obtain the quadrature error as

$\Omega_{z}^{Q}=\frac{\sin (2 n \psi)}{4 n A G} \frac{\left|H_{1}\left(j \omega_{1}\right)-H_{2}\left(j \omega_{1}\right)\right|}{\omega_{1}\left|H_{1}\left(j \omega_{1}\right) H_{2}\left(j \omega_{1}\right)\right|}$

where $H_{1}\left(j \omega_{1}\right)$ and $H_{2}\left(j \omega_{1}\right)$ (with $j$ being the imaginary unit) are the transfer functions associated with the $\{\cos (n(\theta-\psi))\} \quad$ and $\quad\{\sin (n(\theta-\psi))\} \quad$ modes, respectively.

\section{SIMULATIONS}

We consider the VRG structure shown in Figure 1. Its ring has the mean radius $R=1550 \mu \mathrm{m}$ and the thickness $h=35 \mu \mathrm{m}$. We assume $w_{\min }=99.9 \mu \mathrm{m}$ and $w_{\text {max }}=$ $100 \mu \mathrm{m}$ to have a width variation of $100 \mathrm{~nm}$. We consider the mode numbers $n=2,3$ with the quality factor of $q_{f}=10000$. At the nodal points of each mode, identical beams of the width $16 \mu \mathrm{m}$ and length $175 \mu \mathrm{m}$ connect the ring to a circular suspension. The nominal natural frequencies are obtained as $\omega_{0}=61.429 \mathrm{kHz}$ for $n=2$ and $\omega_{0}=142.644 \mathrm{kHz}$ for $n=3$. To model the imperfections in the suspension, we assume that the beam located at $\theta_{1}=0$ has a $1 \%$ width variation. This results in a $15.49 \mathrm{~Hz}$ frequency split for $n=2$. To have a fair comparison, we consider the same frequency split for $n=$ 3 as well. The quadrature error, as a function of the modeshape rotation angle $\psi \in\left[0, \frac{\pi}{2}\right]$, is depicted in Figure 4 . We observe that, for a fixed frequency split, the $n=3$ mode has a larger peak value for the quadrature error, mainly due to lower angular gain. For $n=2$, the quadrature error reaches its maximum at $\frac{\pi}{8}$; this corresponds to the case where the width nonuniformity angle, $\phi$ lies halfway between the peak angles of the $\{\cos (2 \theta)\}$ and $\{\sin (2 \theta)\}$ modes. This maximum is intuitive as the coupling is expected to peak in the midpoint. In terms of the absolute value, the quadrature error repeats itself by every $\frac{\pi}{4}$ rotation of the mode-shapes, and therefore, it shows a $\frac{\pi}{4}$ periodicity with respect to the width nonuniformity angle, $\phi$.

\section{CONCLUSION}

We presented a general method to analyze the

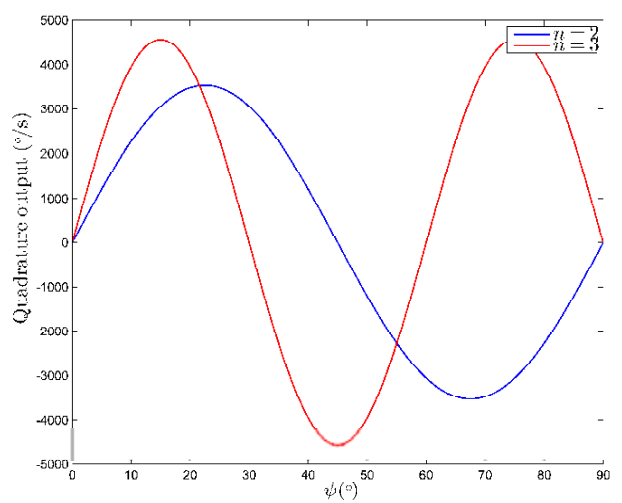

Figure 4: Quadrature error versus the mode-shape rotation angle.

frequency split and quadrature error in VRGs with structural imperfections. Our method can be extended to include more involved imperfection models associated with the etching-related nonidealities (e.g., undercuts) as well as material anisotropy. Effect of different nonuniformity profiles can be found by using our model and superposition which might lead to a higher or lower quadrature. We will pursue these extensions, along with the electrostatic compensation of imperfections, in future research.

\section{ACKNOWLEDGEMENTS}

This work was supported by the Scientific and Technological Research Council of Turkey (TUBITAK) 2232 Program with the grant number 118C247. The ideas/claims presented here solely belong to authors.

\section{REFERENCES}

[1] M. W. Putty, "A micromachined vibrating ring gyroscope.," Ph.D. Dissertation, University of Michigan, 1995.

[2] B. J. Gallacher et al., "Electrostatic correction of structural imperfections present in a microring gyroscope," J. Microelectromechanical Syst., vol. 14, no. 2, pp. 221-234, Apr. 2005.

[3] P. A. A. Laura et al., "Vibrations of rings of variable cross section," Appl. Acoust., vol. 25, no. 4, pp. 225234, Jan. 1988.

[4] P. Bisegna and G. Caruso, "Frequency split and vibration localization in imperfect rings," J. Sound Vib., vol. 306, no. 3-5, pp. 691-711, Oct. 2007.

[5] J. Liu et al., "Estimation and elimination of eigenvalue splitting and vibration instability of ringshaped periodic structure subjected to three-axis angular velocity components," Meccanica, vol. 54, no. 15, pp. 2539-2563, Dec. 2019.

[6] S. S. Rao, Vibration of continuous systems. Hoboken, N.J: Wiley, 2007.

[7] T. Charnley et al., "Vibrations of thin rings of rectangular cross-section," J. Sound Vib., vol. 134, no. 3, pp. 455-488, Nov. 1989.

[8] R. Eley et al., "Coriolis coupling effects on the vibration of rotating rings," J. Sound Vib., vol. 238, no. 3, pp. 459-480, Nov. 2000.

\section{CONTACT}

*M. Hosseini-Pishrobat, tel: +90-312-2901219; mehran@ee.bilkent.edu.tr 